\title{
Atmospheric Energy and Variable Sleep Cycle based on Wireless Sensor Network Design
}

\author{
Xuxiu $^{1,2}$ \\ ${ }^{1}$ School of computer science and Technology,CUMT. JiangsuXuzhou 221116 \\ 2 Henan Yima Coal Group ,HeNanYima 472300 \\ ${ }^{1,2}$ xuxiu@cumt.edu.cn,
}

Keywords: wireless sensor, variable sleep, ZigBee .

Abstract. According to the atmospheric information collection system has its own characteristics, to design a collection for long uninterrupted data transmission packet intermittent, low-power wireless sensor networks, and can automatically identify low energy equipment, restructuring forwarding path to extend the life of the network, improve data collection efficiency.

\section{Introduction}

Air data collection methods have intermittent, predetermined, long-term, so the wireless sensor network mainly in the energy and validity of the connection request.

Low-power indicator is one of the characteristics zigbee protocol, is a big advantage compared to other wireless protocols, in order to further reduce power consumption zigbee devices, combined with intermittent air data collection methods, and should make zigbee devices in the non-working period goes into sleep mode but during sleep the device can not communicate only when the device transitions from the sleep mode to work mode can communicate with other devices.

So the exact make zigbee device from Sleep to start is one of the factors involved in this article. For the validity of the connection request, consider the use of energy-based routing protocols zigbee network optimization designed to prevent a full-featured child node node node failure because of the Father and the connection is lost, resulting in part of the network data generated empty.

\section{Zigbee Introduction}

Zigbee is a new short-range, low-power, low data rate, low complexity, strong anti-interference wireless sensor networks. Medical, military, intelligent home, industrial control, logistics and other fields, more and

more attention. ZigBee protocol stack, physical layer and MAC layer using IEEE802.15.4 standard, the network layer specification developed by the ZigBee Alliance. Since the energy ZigBee network node is limited, so select the network layer routing strategy for the entire sensor network plays a vital role. Zigbee protocol stack consists of a physical layer, MAC layer, network layer, security layer specification and high-level application components. This paper discusses the organizational structure of the network layer: the network layer are nodes join or leave the network, storage-related information forneighbors,routing route discovery maintenance.And other functions, support for the star, tree , grid (Mesh), and other topologies.

\section{Tree Routing Protocol (TR)}

Tree routing TR (Tree Routing) is divided into two parts: tree tree address negotiation and routing. When the coordinator to establish a network, it uses its own address as the address 0 , the definition of its own network depth Depth0 $=0$. If the node $\mathrm{i}$ want to join the network and establish contacts with node $\mathrm{k}$, the node $\mathrm{k} \mathrm{i}$ become a parent. Then assign node $\mathrm{i}$ and network address Ai depth Depthi $=$ Depthi +1 . Depth is from the root node network (coordinator) to jump the node series. 
A new node $\mathrm{n}$ is an RFD (simple function device), then it has no routing capabilities. It wants to join the network, the network based on its depth $\mathrm{d}, \mathrm{k}$ parent is going to give it to assign addresses: $\mathrm{An}=\mathrm{Ak}$ + Cskip (d) $* \mathrm{Rm}+\mathrm{n}$; If the new node is a full function device (FFD), then it has the routing function. Parent node $\mathrm{k}$ to be allocated to address it:An $=\mathrm{Ak}+1+$ Cskip $(\mathrm{d}) *(\mathrm{n}-1)$, wherein:

Cskip $= \begin{cases}1+\mathrm{C}_{\mathrm{m}} *\left(\mathrm{~L}_{\mathrm{m}}-\mathrm{d}-1\right), \mathrm{R}_{\mathrm{m}}=1 & \\ \frac{1+\mathrm{C}_{\mathrm{m}}-\mathrm{R}_{\mathrm{m}}-\mathrm{C}_{\mathrm{m}} * \mathrm{R}_{\mathrm{m}}{ }^{\mathrm{Lm}-\mathrm{d}-\mathrm{l}}}{1+\mathrm{R}_{\mathrm{m}}} & , \mathrm{R}_{\mathrm{m}}>1\end{cases}$

Number $\mathrm{Cm}$ represents a router or coordinator biggest child node, $\mathrm{Rm}$ represents a router or coordinating sub-node maximum number of routers, $\mathrm{Lm}$ is the maximum depth of the network.

Suppose a router sends a packet to a destination node address D, its network address and depth are A and $\mathrm{d}$. The router first determines whether the destination node is its child nodes, according to A $<\mathrm{D}$ $<$ Cskip (d-1), if it is a child node, the address of the next hop is $\mathrm{N}$ :

$$
\mathrm{N}=\left\{\begin{array}{c}
\mathrm{D}, \text { end device } \\
\mathrm{A}+1+\left[\frac{\mathrm{D}-(\mathrm{A}+1)}{\text { *Cskip }(\mathrm{d})^{-}}\right] \begin{array}{c}
\text { Cskip }(\mathrm{d}) \\
\text { (otherwise) }
\end{array}
\end{array}\right.
$$

\section{Local repair tree routing (LRTR) sensor network}

LRTR have three valves minimum energy value ER, EE, EW and a constant value E,, where ER represented as router nodes $(\mathrm{ZR})$ and the start of a data transfer; EE representatives terminal device (ZED) work minimum energy requirements; EW Representative issued a warning of low energy consumption which is equal to $48 * \mathrm{EE}$; E, represents power consumed per unit time sleep.

When routing node $\mathrm{i}(\mathrm{ZR})$ energy $\mathrm{Ei}<\mathrm{EW}$ when it sends in each transmission cycle to the parent node low energy warning message, the parent will be uploaded to the root node of the message sequence, suggesting that the central gathering device in a low power state.

Assuming the current data acquisition cycle time $t$, when the routing node energy $2(\mathrm{EE}+\mathrm{t} * \mathrm{E}),<\mathrm{Ei}$ $<\mathrm{ER}+\mathrm{t} * \mathrm{E}$, when it reassigned packet address to the parent node sends a request to its parent again he's being given a RFD address. If it does not have any child nodes, which later joined the network in order to respond to any request for its parent node. Only as a terminal device, no longer has the routing function. If it has a child node, it is to all its child nodes send packets to show their energy has been insufficient as a ZR, after the child node receives the data packet will be re looking for a new parent to rejoin the network. Child node re looking for a new parent process, the child nodes continued to within its communication range sends a request to join the network packets when other router node receives these requests, if the $\mathrm{Cm}, \mathrm{Rm}, \mathrm{Lm}$ constraints , then assign a new address to the requesting node.

When the router nodes i energy $\mathrm{Ei}<2(\mathrm{EE}+\mathrm{t} * \mathrm{E})$, when it sent to its parent node failure packets from the network. Similarly to its child nodes send packets show their lack of energy, let the child nodes to find a new parent to join the network.

As shown in picture 1, When R1 below the warning sent its first energy ID location marked lack of energy to the coordinator, and then send the packet to its child nodes CE and CR express their energy is not sufficient as $\mathrm{ZR}$ nodes. $\mathrm{CR}$ and $\mathrm{CE}$ after receiving the packet, to within its communication range sends a request to join the network packets. Assume that the R2 within communication range of CR, Rl, upon request, will be assigned to a new address for the CR: ACR $=$ AR $1+1+$ Cskip (d) * (n-1). 


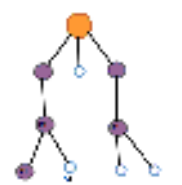

Figure1. R1 energy shortage

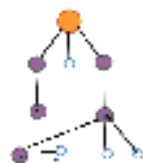

Figure 2. CR R2 access networks

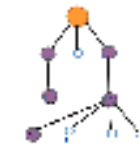

Figure 3 .CE, CR have access R2

$\mathrm{CR}$ after joining the network, if the $\mathrm{CE}$ is not a member $\mathrm{R} 2$ network, the $\mathrm{CR}$ has received $\mathrm{CE}$ join the network request to the $\mathrm{CR}$ as $\mathrm{CE}$ parent node to $\mathrm{CE}$ assign an address to: $\mathrm{ACE}=\mathrm{ACR}+1+\mathrm{Cskip}(\mathrm{d})$ $* \mathrm{Rm}+\mathrm{n}$, the formation of the network shown in Figure 2; If the CE joined the R2 network, (If Cm, $\mathrm{Rm}$, Lm constraint satisfaction) obtained the CE address, ACE $=\mathrm{AR} 2+1+$ Cskip (d) $* \mathrm{Rm}+\mathrm{n}$, the network shown in Figure 3; d on behalf of the parent node of the network according to their energy and depth R1 EE relationship is determined as a parent node failure or terminal device (ZED).

\section{Based On Variable Cycle Data Acquisition}

\section{Sensor Network Clock Synchronization}

Atmospheric sensor network to collect data required to have at the same time, but also need to wake up periodically sleep in order to ensure the reliability of the node on the transmission link.

On the communication relationship, the distinction between the master clock and the clock from the clock, according to PTP best master clock algorithm automatically selects the master clock within the region, from the clock synchronization to keep the slave clock with the master clock.

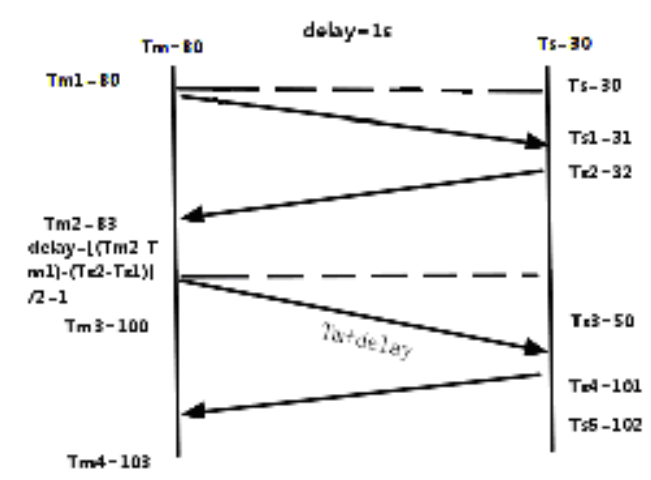

Figure 4: CTS Synchronized Schematic
Based on the above paper, CTS clock synchronization system to achieve optimal synchronization effect.Offset measurement: Since node ZigBee network earlier in this article are left at energies below $2(\mathrm{EE}+\mathrm{t} * \mathrm{E}$,), to the parent node sends leave message, so the use of multicast send each cycle Master way synchronization message, the terminal receives this synchronization packets processed after their time information (including packet processing time, as five Ts1 Ts2) sent to the corresponding master, master received after this time information can calculate the clock offset and the terminal.

(2) Delay measurements: After the synchronization message transmission, master no longer sends delay request message, it is because the use of the offset measurement time information can be calculated to a child node master transmission delay. The method to calculate the delay here and PTP protocols, transmission time master records synchronization packets and response packets of the reception time, the calculated time interval 2 , then subtracting the child node package processing time, as shown in Figure 4, Ts2 Ts1, depending on the network is symmetrical network, and then divided by 2 to obtain a delay time from the clock and the master clock. Then master at this time + delay to send to a terminal node, the node synchronous clock to achieve master-slave synchronization clock.

\section{Variable Sleep Cycle Transport Protocol Design}

According to the atmospheric sensing data needs, a protocol is proposed to change the sleep period to reduce power consumption of the data acquisition.

\section{Network Convergence Time Parameter Determination}

Sleep problems fixed week is facing sensor networks into hibernation after a long, how long it takes to wake before transmitting data. These issues are before the problem sensor transmission network This section focuses on the first time to be synchronized to determine the cycle time, so for the first time before the network goes to sleep well these times need to determine the parameters. Phase synchronization node in the network cycle calculates completion time Tsi network synchronization and data transfer after the start time Tti network, all the nodes to the root node transmits data packets, while the root node synchronization to child clock, each node received or sent the first time a data 
packet is set to T1 to end clock synchronization or data transfer is completed last packet time T2, when the length of the data acquisition $\mathrm{T} 3$, the node working time is $\mathrm{T}=\mathrm{T} 2-\mathrm{T} 1+\mathrm{T} 3$, set the sleep time period to:

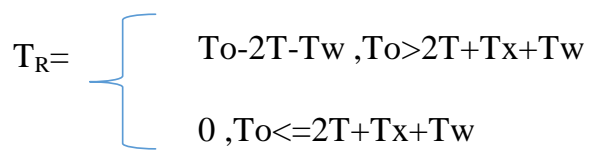

Wherein the desired routing node connection node Tw newly added time, is a constant value, non-routing node is equal to $0, \mathrm{Tx}=\mathrm{Eo} / \mathrm{E}$ '; Eo represents the energy consumed by node startup and shutdown,E'represents dormant consumption per unit of time energy of.

Once a node to determine the time parameters, or the new access node (all the root node to this node)

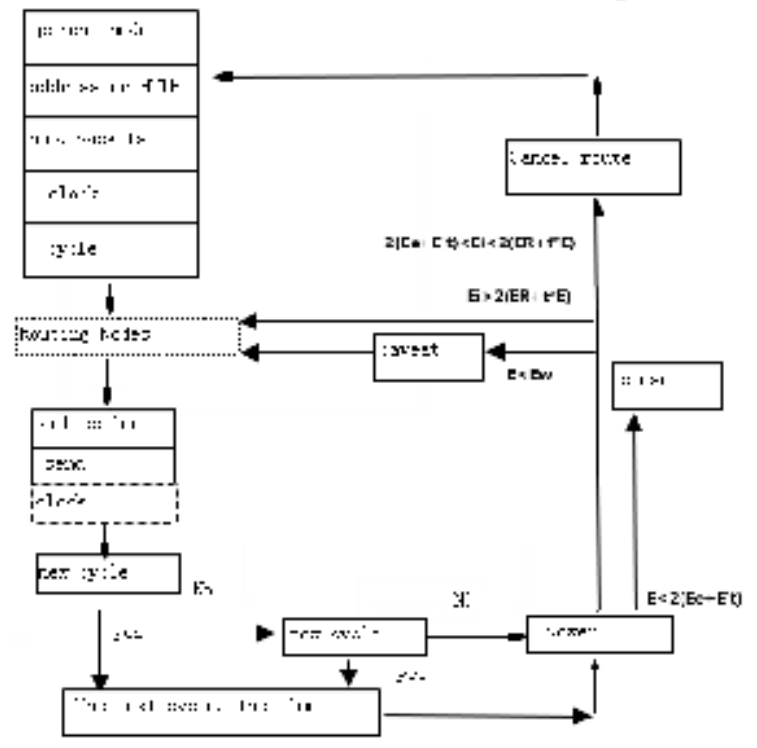
until the next periodic update was recalculated has been used to calculate the parameters between this and the first VCTP process conditions, time, and the parent node synchronization is complete.

\section{Network Sleep Cycle Update}

When the root node receives periodic updates will notice changes after the start of the next cycle of the clock synchronization is complete network update changes, update cycle packet contains a starting time period $\mathrm{Tb}$ and To, to receive the updated node recalculates the time parameters, start-up time and closing time. If the node TR $>0$, then set the start time: $\mathrm{Ts}=\mathrm{Tb}-\mathrm{T}+\mathrm{nTo}$, set off time is: $\mathrm{Te}=\mathrm{Tb}+\mathrm{nTo}+2 \mathrm{~T}$.If the node $\mathrm{TR}=0$, then the node has started. At this point the entire content network cycle is completed.

Figure 5. Sensor Networks To Follow The Work Order

\section{Conclusion}

Atmospheric pollution has become a hot issue, and timely monitoring of the atmospheric environment, air quality is imperative to understand the problem, this paper explore the characteristics of wireless sensor networks monitoring the atmosphere, providing a low-power network protocol designed to extend the wireless network lifetime. The next step will be the time parameter node further optimized to cope with more stringent design requirements.

\section{Acknowledgements}

This work was financially supported by the "China Postdoctoral Science Foundation funded project 2015 T80773 Principal funding";" China Postdoctoral Science Foundation funded project 2013M540572 First funding"

\section{References}

[1] ZigBee Aliance.Network Layer Specification 1.0 [S] .2010 (12).

[2] Li Tao, Yang Jing, Wang Ping based on energy considerations ZigBee tree routing protocol [J], Computer Engineering and Applications, 2012,45: 115-117

[3] EIDSON JC, FISCHER M C, WHITE J. IEEE - 1588TM standard for a precision clock synchronization protocol for networked measurement and control system s [J] .2013.

[4] IEEE Standard.1588-2002, IEEE standard for a precision clock synchronization protocol for networked measurement and control systems [S] .2012. 
[5] Zhang Yan, Sun Hexu IEEE- 1588 in real-time industrial Ethernet Application [J] Microcomputer Information, 2013,21 (9): 19-21

[6] Zigbee Alliance.Home Page [EB / OL] .http: //www.zigbee.org,2012. 